\title{
Heinrich Oberreuter
}

\section{Mehr Demokratie wagen?}

\section{Parlamentskritik und Parlamentsreformen in den 60er und 70er Jahren}

„Mehr Demokratie wagen“ zu wollen, war der Leitsatz der Regierungserklärung Willy Brandts 19691. Der erste sozialdemokratische Kanzler der Bundesrepublik verstand, wie er kurz zuvor in einem Aufsatz dargelegt hatte, Demokratie als „ein Prinzip, das alles gesellschaftliche Sein der Menschen beeinflussen und durchdrin-

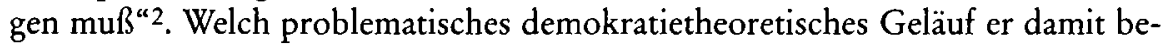
treten hatte ${ }^{3}$, kann Brandt nicht gänzlich bewußt gewesen sein. Tatsächlich meinte er auch nur mehr Mitbestimmung und Mitverantwortung der Bürger und hatte sich nur dem damals üblichen unpräzisen Begriffsgebrauch angepaßt. Auch mit dem zweiten Schlüsselbegriff „Reform“ - der meist gebrauchte in dieser Regierungserklärung ${ }^{4}$ - sollte der Anschein einer Neugründung 5 der Republik erzeugt werden. Brandt verstand aber darunter nur Verbesserungen, nicht grundlegende Veränderungen, also alles andere als einen Umsturz.

Seit 1968 hatte speziell im Kontext der Notstandsgesetzgebung ${ }^{6}$ der Geist der außerparlamentarischen Opposition die Stufen des Bundeshauses umspült wie das Rheinhochwasser. Kritik von außen, lang dauernde interne Diskussionen und vor allem die offene Machtfrage am Ende der Großen Koalition und kurz vor den Wahlen, welche die Frage nach ihrem parteipolitischen Nutzen neutralisierte, ermöglichten 1969 endlich eine umfassendere Parlamentsreform ${ }^{7}$, die korrigierende

1 Deutscher Bundestag, Sten.Ber., 6. Wahlp., 5. Sitzung vom 28. 10. 1969, 20-34.

2 Willy Brandt, Die Alternative, in: Die Neue Gesellschaft 16 (Sonderheft vom 1.5. 1969) 4.

3 Vgl. neben anderen Wilbelm Hennis, Demokratisierung: zur Problematik eines Begriffs (Opladen 21972).

4 Wolfgang Bergsdorf, Herrschaft und Sprache (Pfullingen 1983) $231 \mathrm{ff}$.

5 Wolfgang Jäger, Die Innenpolitik der sozial-liberalen Koalition 1969-1974, in: Karl-Dietrich Bracher, Wolfgang Jäger, Werner Link, Republik im Wandel 1969-1974. Die Ära Brandt (Geschichte der Bundesrepublik Deutschland 5/I, Stuttgart, Mannheim 1986) $15 \mathrm{ff}$.

${ }^{6}$ Heinrich Oberreuter, Notstand und Demokratie. Vom monarchischen Obrigkeits- zum demokratischen Rechtsstaat (München 1978) bes. $218 \mathrm{ff}$.

7 Dazu: Hans Maier, Heinz Rausch, Emil Hübner, Heinrich Oberreuter, Parlament und Parlamentsreform. Zum Selbstverständnis des fünften Deutschen Bundestages, Bonn 1969 (München 21979); Uwe Thaysen, Parlamentsreform in Theorie und Praxis. Zur institutionel- 
Verbesserungen mit sich brachte, aber keine grundlegenden Veränderungen. In seiner Reaktion auf die radikale Systemkritik lag der Bundestag auf der gleichen Linie wie die Regierung und die Gesellschaft insgesamt: Die Revolte blieb draußen vor der Tür. Es bestanden zwei Diskursgemeinschaften, die wenig miteinander gemein haben konnten. Denn die außerparlamentarische Opposition (APO) erstrebte keinen Dialog mit dem Parlament, sondern dessen Überwindung. Anders war dies in den 80er Jahren, als die „Antiparteien-Partei“ der Grünen mitten im Parlament saß. Deren Zähmung ist dann ein Produkt der 90er Jahre gewesen ein Nachweis für die Integrationskraft des parlamentarischen Systems.

Auf diesem Hintergrund sollen Kritik (I), Praxis (II) und Reformbilanz des Bundestages (III) in den 60er und 70er Jahren untersucht werden.

\section{Kritik}

Die meiste Kritik am Parlamentarismus war damals wie heute nicht gleichermaßen differenziert wie die parlamentarische Wirklichkeit. Sie lebte aus historischen Mißverständnissen, Fiktionen, Stilisierungen und Idealisierungen. Durchaus auf Rousseaus Schultern stehend, pflegte sie Vorbehalte gegen das Prinzip der Repräsentation und gegen den Pluralismus. Insoweit hing sie partiell identitätsdemokratischen oder jakobinischen Vorstellungen an. Drei Richtungen lassen sich unterscheiden:

1) Immerhin haben sich als Antwort auf die Herausforderungen der Systemkritik in dieser Periode auch die „kritischen Freunde" 8 des Parlaments formiert. Sie unterzogen diese Institution reformorientierter funktionaler Analyse, wobei ihre Diskussionsbeiträge vor allem die fachwissenschaftliche Öffentlichkeit und die nicht allzu große Gruppe der an Reformen interessierten Parlamentarier erreich-

len Lernfähigkeit des parlamentarischen Regierungssystems. Eine empirische Analyse der Parlamentsreform im 5. Deutschen Bundestag (Opladen 1972); Michael Hereth, Die Reform des Deutschen Bundestages (Opladen 1971). Zu Funktions- und Reformproblemen des Bundestages verfaßten einige Parlamentarier wichtige Beiträge: Hans Apel, Der deutsche Parlamentarismus (Reinbek bei Hamburg 1968); Ulrich Lobmar, Das Hohe Haus. Der Bundestag und die Verfassungswirklichkeit (Stuttgart 1975); Friedrich Schäfer, Der Bundestag. Darstellung seiner Aufgaben und seiner Arbeitsweise. Verbunden mit Vorschlägen zur Parlamentsreform (Köln, Opladen 1967). Vgl. auch die Beiträge in Emil Hübner, Heinrich Oberreuter, Heinz Rausch (Hrsg.), Der Bundestag von innen gesehen (München 1969) sowie Winfried Steffani (Hrsg.), Parlamentarismus ohne Transparenz (Opladen 1971) und Heinz Rausch, Parlamentsreform - Tendenzen und Richtungen, in: ZfP 14 (1967) 259ff; Emil Hübner, Die Beziehungen von Bundestag und Bundesregierung im Selbstverständnis der Abgeordneten des V. Deutschen Bundestages (München 1980). Eine präzise, knapp umrissene Gesamtschau bietet Ulrich Scheuner, Zur Entwicklung des Parlaments unter der Ordnung des Grundgesetzes, in: Gerhard A. Ritter (Hrsg.), Gesellschaft, Parlament und Regierung. Zur Geschichte des Parlamentarismus in Deutschland (Düsseldorf 1974) 421-441.

8 Walter Euchner, Zur Lage des Parlamentarismus, in: Gert Schäfer, Carl Nedelmann, Der CDU-Staat (München 1967) 69. Vgl. auch Thaysen, Parlamentsreform (wie Anm. 7) 59. 
ten ${ }^{9}$. Ihre Forderungen und Ansätze fanden ihre Zusammenfassung in jenem multidimensionalen Optimierungsmodell, welches Winfried Steffani Anfang der 70er Jahre entwickelt hatte ${ }^{10}$. Postuliert wurde zum einen die verstärkte Wahrnehmung der Öffentlichkeitsfunktionen, deren Vernachlässigung trotz frühzeitiger Warnungen mit einer sektoralen Legitimitätskrise quittiert werden mußte, zum anderen der Ausbau der technisch-wissenschaftlichen Infrastruktur ${ }^{11}$, um das Parlament im raschen Wandel von Quantität, Qualität und Komplexität der sich ihm stellenden Aufgaben wettbewerbs- und steuerungsfähig zu halten. Zunehmend wurde als dritte Aufgabe auch die Angleichung von Strukturen und Verfahren an den Typus des parlamentarischen Regierungssystems bewußt. Diese funktionale Kritik geht auch normativ von diesem Typus aus und orientiert Reformen an der Sicherung seiner Funktionsfähigkeit in der Zukunft.

2) Der modernitätspessimistische Zweig der Kritik ist geradezu durch seine Perzeptionsverweigerung der Strukturprinzipien des parlamentarischen Regierungssystems charakterisiert. Parlamentarische Parteiregierung, Gewaltenfusionierung und checks and balances, Partei- und Fraktionsbindungen des Abgeordneten, Arbeitsteilung und Spezialisierung, Ausdifferenzierung der Willensbildung und vor allen Dingen der Einfluß gesellschaftlicher Interessen gelten ihm als Verfallsphänomene. Wo sich Substanz und Funktion gewandelt haben, wird ohne genauere empirische Analyse Substanzentleerung und Funktionsverlust konstatiert. Ernst Fraenkel hat frühzeitig diese landläufige Kritik als das Kritikbedürftigste am Bonner Parlamentarismus bezeichnet: „Sie ist reaktionär und schizophren. Sie sehnt sich heimlich nach einer starken Regierung und bekennt sich öffentlich zu der Herrschaft eines allmächtigen Parlaments. Sie beschimpft den Abgeordneten, wenn es zu einer Regierungskrise kommt, und verhöhnt ihn, wenn er getreulich die Fraktionsparole befolgt. Sie verkennt die notwendigerweise repräsentative Natur eines jeden funktionierenden Parlamentarismus und verfälscht seinen Charakter, indem sie ihn plebiszitär zu interpretieren versucht."12

Das Verfallsparadigma wurde bekanntlich frühzeitig von Carl Schmitt formuliert ${ }^{13}$. Sich besonders auf französische Theoretiker aus dem Vormärz berufend, definiert Schmitt Diskussion und Öffentlichkeit als die geistigen Grundlagen des Parlamentarismus. Gemeint ist damit die Suche nach Wahrheit in öffentlicher Diskussion durch parteipolitisch und interessenspezifisch nicht gebundene Abgeord-

${ }^{9}$ Ein Zentrum war und ist die 1970 gegründete Deutsche Vereinigung für Parlamentsfragen mit der von ihr herausgegebenen Zeitschrift, die für die politische und wissenschaftliche Orientierung unerläßlich geworden ist.

10 Winfried Steffani, Parlamentarische Demokratie - Zur Problematik von Effizienz, Transparenz und Partizipation, in: ders. (Hrsg.), Parlamentarismus ohne Transparenz (Opladen 21973) 17-47.

11 Helmut Quaritsch, Die wissenschaftlichen Dienste des Deutschen Bundestages, in: Festschrift für Ernst Forsthoff (München 1972) 303-324.

${ }^{12}$ Ernst Fraenkel, Strukturdefekte der Demokratie und deren Überwindung, in: ders., Deutschland und die westlichen Demokratien (Stuttgart u.a. [erstm.] 1964) 56.

${ }^{13}$ Carl Schmitt, Die geistesgeschichtliche Lage des heutigen Parlamentarismus (Berlin [erstm.] 1923, 41969). 
nete. Daran läßt er die Wirklichkeit des modernen Parlamentarismus in der Massendemokratie scheitern. Kompromisse lehnt er als Widerspruch zu dessen Wesen ab. Carl Schmitt hatte mit diesen Thesen der rechten Parlamentarismus- und Pluralismuskritik in Weimar ihre Argumente geliefert. Unter ausdrücklicher Berufung auf diese Thesen ${ }^{14}$ und in deutlicher Anlehnung an sie soufflierte in den 60er Jahren Jürgen Habermas der neuen Linken ihre kritischen Stichworte, so vergröbert sie dort auch aufgenommen worden sein mochten. Auch Habermas geht es um Wahrheit und Vernunft, die sich in einem ständigen diskursiven Prozeß zum allgemeinen Konsens formen sollten, um Herrschaft in Vernunft aufzuheben. Abweichungen von diesem Idealbild fänden ihren Grund in der antagonistischen Struktur der bürgerlichen Gesellschaft. Wenn im parlamentarischen Prozeß nicht Wahrheit gesucht, sondern Interessen ausgehandelt und Kompromisse gebildet werden, dann gilt dies als Abirrung vom "ursprünglichen“ und „eigentlichen“ Parlamentarismus. Zusätzlich hat Habermas das Parlament aus der Einheit und Kontinuität der politischen Kommunikation verabschiedet und sah die kritische Funktion seiner Publizität zu einer demonstrativen herabgesunken. Als Beleg dafür diente ihm nicht zuletzt auch die Leibholzsche Parteienstaatslehre ${ }^{15}$ - eine höchst anfechtbare Doktrin, die als empirischer Nachweis für weitreichende Thesen gänzlich untauglich ist.

Pluralistische Demokratie beruht weder auf der Homogenität der Interessen, noch kann sie sich prozedural als Modus der Wahrheitssuche definieren. Auch der modernen offenen Gesellschaft sind solche Annahmen fremd. Schmitt wie Habermas verurteilten durch ihre normativen Postulate den modernen Parlamentarismus zum Scheitern, ganz abgesehen davon, daß auch ihr Leitbild historisch nie Realität, sondern immer nur Theorie oder literarische Fiktion gewesen ist. Vernunft und Wahrheit stehen bei beiden im Mittelpunkt, wenn für Carl Schmitt ohne Unabhängigkeit von Parteien und Interessen Diskussion nicht wahr und infolgedessen Öffentlichkeit nicht vernünftig sein kann ${ }^{16}$, und wenn Habermas dementsprechend "das deliberierende Parlament als Mitte" politischer Kommunikation postuliert ${ }^{17}$. Die Krise des modernen Parlamentarismus wird zu einem unentrinnbaren Dauerzustand, da seine Wesensbestimmung ja gerade darin liegt, Konflikt und Konsens der sozialen und politischen Interessen zu kanalisieren und ein Mindestmaß gesellschaftlicher Integration zu bewirken. Wenn allerdings für die politisch-parlamentarische Kommunikation das kritische Prinzip geradezu normativ überhöht, Integration dagegen gegeißelt wird, gibt unter der Hand eine moderne Aufgabenerfüllung unvermittelt Anlaß zur Fundamentalkritik. Inwiefern Integration a priori des kritischen Prinzips enträt und inwieweit moderne, hochdifferenzierte und großräumige Gesellschaften ohne Integrationsprozesse

14 Jürgen Habermas, Strukturwandel der Öffentlichkeit (Neuwied, Berlin ${ }^{21965)}$ 94. Ausführlich Hartmuth Becker, Die Parlamentarismuskritik bei Carl Schmitt und Jürgen Habermas (Berlin 22003) bes. $149 \mathrm{ff}$.

15 Gerbard Leibholz, Der Strukturwandel der modernen Demokratie (Karlsruhe ${ }^{31967) .}$

16 Carl Schmitt, Verfassungslehre (Berlin 1928) 319.

17 Habermas, Strukturwandel (wie Anm. 14) 225. 
überlebensfähig wären, bleiben ungestellte und unbeantwortete Fragen. Allerdings ist in einem homogenitätsbestimmten Gesellschaftsmodell Integration a priori kein Thema, da sie vorausgesetzt wird.

Auf die Herkunft dieses wirkmächtigen Idealbildes aus frühliberalen, vor allem französischen Parlamentarismuskonzepten samt deren Ablehnung von Parteien und Interessenvertretung ist vielfach aufmerksam gemacht worden ${ }^{18}$. Tatsächlich aber sind Parlamente stets auch Arenen für Politik- und Interessenpluralismus gewesen. Selbst Edmund Burke hat in seiner berühmten Rede an die Wähler von Bristol am 3. November 1774 nicht nur die Gewissensfreiheit der Abgeordneten, sondern auch die vielschichtigen Interessen beschworen, die erwogen und ausgeglichen werden müssen ${ }^{19}$.

3) Die radikale Systemkritik ging über die von Carl Schmitt und Jürgen Habermas begründeten Legitimitätszweifel gegenüber einem Parlamentarismus, der seine geistigen Grundlagen und seine moralische Wahrheit verloren habe, hinaus. Sie erstrebte nichts weniger als seine Überwindung. Aber in nuce hatten beide Autoren das kritische Arsenal der außerparlamentarischen Opposition bereitgestellt. Johannes Agnoli, der es zugespitzt und popularisiert hat ${ }^{20}$, stand theoretisch und methodisch auf beider Schultern. Er teilte auch ihre empirischen und historischen Defizite, auf deren Grundlage sich parlamentarischer Funktionswandel als Funktionsverlust ausweisen ließ, während dieser Wandel doch tatsächlich einen erheblichen Funktionsgewinn darstellte. Allein solche Empirieferne kann die These begründen, das parlamentarische Regierungssystem entstamme einer bestimmten historischen und gesellschaftlichen Epoche und diene entweder der Erhaltung überholter Verhältnisse oder sei mit diesen zum Untergang verurteilt. Dieses System wird dabei - begrifflich unhaltbar - mit dem konstitutionellen Parlamentarismus und seinen dualistischen Grundannahmen identifiziert. Demgemäß werden Parlament und Regierung wieder auseinandergerückt, um die Basis für die Behauptung zu gewinnen, das Parlament legitimiere nur noch die Herrschaft außerparlamentarischer Oligarchien, zu denen ganz unbefangen auch die parlamentarische Regierung gerechnet wird. Agnoli beklagt den Untergang der klassischen parlamentarischen Demokratie und die Überwindung der klassischen parlamentarischen Qualität des frühen bürgerlichen Staates, die von der politischen wie legislatorischen Vormacht des Parlaments gegenüber der Exekutive gekennzeichnet gewesen seien ${ }^{21}$. Tatsächlich gibt es dafür aber keine historische Entsprechung. Auch der

18 Vgl. dazu Gerbard A. Ritter, Der Antiparlamentarismus und Antipluralismus der Rechtsund Linksradikalen, in: Der Überdruß an der Demokratie (Köln 1970) 43-91; ders., Direkte Demokratie und Rätewesen in Geschichte und Theorie, in: Erwin K. Scheuch (Hrsg.), Die Wiedertäufer der Wohlstandsgesellschaft (Köln 1968) 188-216; Wolfgang Jäger, Öffentlichkeit und Parlamentarismus. Eine Kritik an Jürgen Habermas (Stuttgart u.a. 1973). Vgl. aber auch Kurt Lenk, Wie demokratisch ist der Parlamentarismus? (Stuttgart u.a. 1972).

19 Edmund Burke, The Works. A New Edition, Bd. 3 (London 1808) 21.

20 Johannes Agnoli, Peter Brückner, Die Transformation der Demokratie (Frankfurt a.M. 1968).

21 Johannes Agnoli, Thesen zur Transformation der Demokratie und zur außerparlamentarischen Opposition, in: Neue Kritik 47 (1968) $24 \mathrm{ff}$. 
Rekonstruktionsversuch eines Modells politischer Kommunikation ohne Vermittlungsprozesse leidet empirische Not. Sein Vorbild findet sich höchstens im bürgerlich-plutokratischen Parlamentarismus, als vielleicht ein Dutzend gebildeter und vermögender Bürger als Nominierungs- und Wahlkörperschaft für ihren Vertreter zusammentrafen und Repräsentation und Teilhabe an der Herrschaft praktisch in eins fielen ${ }^{22}$. Herrschaft war ohnehin der Scheidebegriff der Auseinandersetzung. Denn Legitimation von Herrschaft kann nur als Dekadenz verurteilen, wer Herrschaft an sich ablehnt und Demokratie als Selbstherrschaft des Volkes im Wortsinn, aber nicht als legitime, verantwortliche und rechtsstaatlich eingeengte Herrschaftsordnung begreift. Dieser Stoß richtete sich schlechthin gegen die repräsentative Demokratie, die durch eine plebiszitäre ersetzt werden sollte.

Im Rahmen seiner kritisch konstatierten Dekadenz nahm das Parlament nach Agnoli ${ }^{23}$ drei Aufgaben wahr: die „Konstitutionalisierung“ politischer Entscheidungen, die "friedlich-manipulative Integration" und die "Veröffentlichung von Herrschaft". Funktions- und Leistungsfähigkeit dabei wird ihm ausdrücklich bescheinigt, ja gigantisch übersteigert; denn darauf beruht die Verschwörungshypothese, nach der die Legitimationsmechanismen des Parlamentarismus dazu dienen, das Bewußtsein der Massen zu ungunsten ihrer objektiven und primären Interessen zu manipulieren. Der Parlamentarismus wäre demnach genau dort am leistungsfähigsten gewesen, wo empirisch orientierte funktionale Kritik bedenkliche Defizite seiner Legitimationsleistung geortet hatte: bei den Öffentlichkeitsfunktionen. In einen anderen, „bürgerlichen“ demokratietheoretischen Argumentationskontext übertragen, ergäbe sich im Ergebnis eine atemberaubend unkritische Funktionsbestätigung der repräsentativen Demokratie. Ganz nebenbei dokumentiert sich hier der verfassungshistorische Fortschritt. Der frühe Parlamentarismus und mit ihm das Volkssouveränitätsprinzip hätten nämlich in Deutschland seinerzeit ihren Kampf gewonnen, wäre ihnen die Konstitutionalisierung der Herrschaft gelungen und hätten sie deren Legitimität unter ihre Botmäßigkeit gebracht. Sobald aber Manipulation als Funktion eines jeden denkbaren modernen Herrschaftsprozesses angenommen wird, bleibt diese Einsicht verstellt.

Diese Manipulationsthese nährte sich aus dem marxistischen Grundwiderspruch von Kapital und Arbeit an der gesellschaftlichen Basis. Die „wahren Interessen“ lägen darin, ihn bewußt zu machen. Denn die Austragung von Sekundärkonflikten im parlamentarischen System diene nur der Verschleierung dieses Widerspruchs und wirke damit seiner Auflösung - mithin der fundamentalen Infragestellung des Systems und der Beseitigung von Herrschaft - entgegen ${ }^{24}$.

Als plebiszitäre systemüberwindende Alternative wurde das Rätesystem propagiert. Nach dem von Karl Marx beschriebenen Muster der Pariser Kommune ${ }^{25}$

22 Dazu Hans Setzer, Wahlsystem und Parteienentwicklung in England, Wege zur Demokratisierung der Institutionen 1832-1948 (Frankfurt a.M. 1973).

${ }^{23}$ Agnoli, Brückner, Die Transformation (wie Anm. 20) $62 \mathrm{ff}$.

24 Ebd. 19 ff.

${ }^{25}$ Karl Marx, Der Bürgerkrieg in Frankreich, in: Marx-Engels Studienausgabe, Bd. 4 (Frankfurt a.M. 1966) $212 \mathrm{ff}$. 
soll es in überschaubaren Basiseinheiten organisiert und durch lockere Föderation zusammengefaßt sein. Dadurch sollte die Identität von Regierenden und Regierten hergestellt und Selbstbestimmung durch ständige aktive politische Partizipation verwirklicht werden.

Kritikbedürftig sind bereits die demokratietheoretischen Prämissen. Bekanntlich setzt die Identitätsdemokratie die Homogenität der politischen und sozialen Interessen voraus. Die zur Erhaltung dieser Identität notwendige Vorwegbestimmung des Gemeinwohls schließt Meinungskonkurrenz und Interessenpluralität aus - nach Rousseau, auf den diese Theorie zurückgeht, überhaupt jede „dornenvolle Diskussion" 26 im Entscheidungsprozeß, in welchem es nicht mehr um bessere Lösungen, sondern um den Vollzug der Wahrheit geht. Aus dem Homogenitätspostulat entwickelt sich - wie u.a. Gerhard A. Ritter herausgearbeitet hat ${ }^{27}$ als zweite Voraussetzung konsequent "die Ausscheidung oder Vernichtung des Heterogenen "28. Dafür hatten auch damals die modernen Theoretiker (wie auch die Praktiker in den noch bestehenden sozialistischen Volksdemokratien) keine humaneren Mittel anzubieten als Erziehungsdiktatur, Gefängnis und Zwangsexilierung ${ }^{29}$. Ein "wahrer" Volkswille wird uneingeschränkt souverän, sein Durchgriff auf das System, das er sich schafft, totalitär; denn die Identitätsannahme verleitet zur Theorie (aber nicht zur Praxis) der Herrschaftslosigkeit, mit der zugleich jede rechtsstaatliche Begrenzung der Macht dahinfällt.

Das Funktionieren eines auf solchen Voraussetzungen beruhenden Systems im Endstadium illustrierte André Gorz ${ }^{30}$ am Beispiel einer Schuhfabrik, deren Arbeiter in freier kollektiver Selbstbestimmung über Produktion und Konsum befinden, z. B. darüber, ob „vier Paar Schuhe per Einwohner und Jahr, die schnell abgetragen sind, oder ein widerstandsfähiges Paar und zwei Paar, die sich schnell abnutzen" zu produzieren seien. Ohne Zweifel marginalisierte die große Systemalternative das hohe Gut politischer Partizipation und negierte geradezu das Problem der Koordination und Steuerung hochkomplexer Gesellschaften. Jedes Rätesystem wäre daran gescheitert, ohne auch nur im entferntesten die Konkurrenz zur Leistungsfähigkeit des modernen Parlamentarismus aufnehmen zu können.

\footnotetext{
${ }^{26}$ Jean Jacques Rousseau, Contrat Social, Buch III, Kap. 4 und Buch II, Kap. 4.

$27 \mathrm{Vgl}$. Anm. 18.

28 Scbmitt, a.a.O. (wie Anm. 13) 14.
}

29 "Ein Gespräch über die Zukunft" mit Rudi Dutschke, Bernd Rabebl und Christian Semler, in: Kursbuch 14 (1968) 171. Die damalige Exilierung von Systemkritikern aus der Sowjetunion - Solschenizyn, Amalrik u.a. - oder auch aus der DDR - Wolf Biermann, Reiner Kunze - war gerade kein Beweis für Liberalisierung, sondern für ungebrochenen Totalitätsanspruch. In bezug auf die neue deutsche Linke sprach Erwin K. Scheuch, Das Gesellschaftsbild der „Neuen Linken“, in: ders. (Hrsg), Die Wiedertäufer (wie Anm. 18) 120, von der „Endlösung" der Antiautoritären“.

30 André Gorz, Der schwierige Sozialismus (Frankfurt a.M. 1968) 129. Kritisch dazu: Fritz Scharpf, Demokratietheorie zwischen Utopie und Anpassung (Konstanz 1970) bes. 54-65; vgl. u. a. auch Wilfried Gottschalch, Parlamentarismus und Rätedemokratie (Berlin 1968); Eric Ertl, Alle Macht den Räten? (Frankfurt a.M. 1968). Siehe dazu Udo Bermbach, Ansatz zu einer Kritik des Rätesystems, in: Berliner Zeitschrift für Politologie 4 (1968) 21 ff. 


\section{Praxis: Funktionsgemäße Modernisierung}

In der Praxis vollzog sich in den 60er Jahren eine modernisierende Anpassung des Selbstverständnisses und der Arbeitsstrukturen des Parlaments an die aktuellen Herausforderungen, welche die vielen und diffusen, systematisch nie gebündelten und sehr ins Detail gehenden Vorschläge zur Parlamentsreform hinter sich ließ. Diese Anpassung diente im wesentlichen einer Zielsetzung: die Funktionsfähigkeit des Parlaments bei seiner Beteiligung an der politischen Führung zu stärken. $\mathrm{Da} ß$ die Staatsleitung Parlament und Regierung „zur gesamten Hand“ zur Verfügung stehe und daß der Bundestag mitregiere ${ }^{31}$, waren Einsichten, welche die staatsrechtliche Diskussion der systemkritischen voraus hatte.

1) Obgleich damalige Messungen des parlamentarischen Selbstverständnisses zu widersprüchlichen Befunden führten ${ }^{32}$, hatte sich, ausgehend von der schon im Parlamentarischen Rat vorhandenen Einsicht über die Abhängigkeit eines funktionsfähigen Parlaments von einem verantwortungswilligen Parteiensystem, sowie in Verarbeitung der Alltagserfahrungen der erfolgreichen ersten Legislaturperioden, eine praktische Verinnerlichung der Funktionslogik des parlamentarischen Regierungssystems vollzogen. In den 60er und 70er Jahren befand sich der Bundestag längst auf einem gefestigten Pfad zum Parteien-, Fraktions- und Koalitionsparlamentarismus.

Wenn die Verfassung institutionell der Regierung als einzige Legitimationsquelle das Vertrauen des Parlaments (d.h. der Parlamentsmehrheit) zuweist und ihre Verselbständigung im Sinne der obrigkeitsstaatlichen Tradition verhindert werden soll, dann müssen sich zwischen Mehrheitsfraktion(en) und Regierung Kommunikationsstrukturen und Interaktionsmuster dauerhaft etablieren, mit deren Hilfe die Übereinstimmung der politischen Zielvorstellungen ständig hergestellt und erhalten werden kann. Darauf beruht materiell die Legitimität der politischen Führung, aber auch das gerade in der 5. Wahlperiode (1965-69) gewachsene Selbstbewußtsein ihrer Fraktionen. Das parlamentarische Regierungssystem der Bundesrepublik enthält gleichzeitig starke Elemente der Kanzler-, der Kabinetts- und der Mehrheitsdemokratie. So wie die Regierung parlamentarische Parteiregierung ist, so ist die Regierungspolitik Mehrheitspolitik. Daß Parteien sich für die Regierung, die sie bilden, verantwortlich fühlen, war in der Bundesrepublik eine wichtige Korrektur historischer Vorbelastungen, an denen zum Beispiel die Weimarer Republik nicht zuletzt gescheitert war.

31 Ernst Friesenhabn, Parlament und Regierung im modernen Staat, in: VVDSt RL 16 (1958) 9-73, hier 38.

32 Maier u. a., Parlament (wie Anm. 7) bes. 26ff. Die Abgeordneten des 5. Bundestages waren mit dem „britischen“ (regierende Mehrheit vs. Opposition), dem „konstitutionellen" (Parlament vs. Regierung) und einem Modell, welches die enge Bindung von Mehrheit und Regierung, aber auch beider Eigenständigkeit postulierte, konfrontiert werden. Dem „konstitutionellen" Modell gaben nur 27,2\% den Vorzug, 67,9\% hielten es für das wirklichkeitsfremdeste und nur $24,1 \%$ glaubten, es entspreche am ehesten dem Selbstverständnis der Parlamentarier. 
Doch dieser Gewinn hat seinen Preis: Bindungen und politische Abhängigkeiten sind permanent und gegenseitig. Regieren im Dauerkonflikt mit einer nicht fluktuierenden, sondern festgefügten Mehrheit wird undenkbar. Dieser Mehrheit steht praktisch und verfassungspolitisch die Opposition mit einer eindeutigen alternativen Rollenzuweisung gegenüber. Zusätzlich formte die vom Grundgesetz unterstützte Rolle der Parteien als faktisch dominierende politische Aktionseinheiten das parlamentarische System der Bundesrepublik auf nationaler Ebene zu einem $Z$ weigruppensystem mit relativ hoher Geschlossenheit, weil zum einen die politische Sozialisation des Abgeordneten durch die Einbindung in die Partei bestimmt wird und zum anderen die Fraktionen sich durchaus als parlamentarischer Arm ihrer Partei verstehen, wie prekär im einzelnen diese Beziehung sich auch gestalten mag. Die Systemkritik postulierte seinerzeit vehement das imperative Mandat ${ }^{33}$. Speziell in der Sozialdemokratie, noch mehr später bei den Grünen, gab es basisimperative Strömungen, die jedoch zurückgewiesen wurden. Sozialdemokratische Bundeskanzler hielten dem „Parteitagsstaat" 34 ihre Bindung an den Amtsgedanken, also an die Herrschaft als anvertrautes, zum Wohle aller Bürger zu gebrauchendes Amt, entgegen. Diese Differenzierung zwischen Amtsverständnis und Mitgliedschaftsinteresse bedeutet keine grundsätzliche Verweigerung der aus natürlichen Bindungen erwachsenden Solidarität und Loyalität. Sie begrenzt aber die Überwucherung der staatlichen Ämter und Institutionen durch rein parteipolitische Optionen. Innerhalb dieser Grenzen bleibt jedoch das Verhältnis von Partei, Abgeordneten und Fraktion auf Dauer gestellt.

Später, 1989, hat Karlsruhe zwar die Rechte des einzelnen Abgeordneten verteidigt, zugleich aber die Fraktion als politisches Gliederungsprinzip für die Arbeit des Bundestages akzeptiert und verfochten ${ }^{35}$. Das Fraktionenparlament ${ }^{36}$ war in den 60er Jahren bereits ausgeprägt. Dort entfaltet sich der einzelne Abgeordnete im wesentlichen im Rahmen eines arbeitsteilig organisierten Kollektivs. Zugleich stellt auch die Geschlossenheit als Basis der Aktionsfähigkeit nach innen wie nach außen ein hohes Gut dar, das in vielfältig mitbestimmten Kommunikationsprozessen hergestellt und gesichert werden muß. Zwischen Individuum und Gruppe kann Regieren zu erschöpfender Integrationsarbeit werden. Zugleich vermögen aktive Minderheiten unter den Auspizien innerparteilicher Integration auf die Regierungspolitik zumindest dadurch erheblichen Einfluß auszuüben, daß sie Entscheidungen verzögern, verwässern oder auch verhindern. Sobald die Einheit der Partei auf dem Spiel zu stehen scheint, können sie durchaus Berücksichtigungs-

33 Dazu Udo Bermbach, Probleme des Parteienstaates. Der Fall Littmann, in: ZParl 3 (1979) 342-363 und Carl Otto Lenz, „Ist die Freiheit des Abgeordneten veraltet und historisch überholt?", in: ZParl 4 (1970) 478-481.

34 Willy Brandt, Was soll das Gerede vom Parteitagsstaat?, in: Vorwärts vom 13.10. 1977, abgedruckt in: Heino Kaack, Reinhold Roth (Hrsg.), Handbuch des deutschen Parteiensystems, Bd. 1 (Opladen 1980) 310-316.

${ }^{35}$ BVerfGE 80, 188-235.

36 Uwe Thaysen, Parlamentarisches Regierungssystem der Bundesrepublik Deutschland (Opladen 1976) 69ff. 
zwänge schaffen, andererseits aber ebenso Konformitätsdruck unterliegen. Damit bleibt der Grundsatz innerparteilicher Demokratie auch im Fraktionenparlament erhalten - ein Argument gegen voreiligen Oligarchieverdacht. Gerade in der Zeit der Kanzlerschaft Ludwig Ehrhards, noch mehr in der Großen Koalition, fand die Richtlinienkompetenz des Kanzlers ihre Grenzen in dem, was der eigenen Mehrheit oder einer Koalition zugemutet werden konnte. Entscheidend für die Position des Kanzlers ist nicht die Grundgesetznorm, sondern seine Durchsetzungschance im eigenen Lager. Durch die Demokratisierungsdiskussion in der Gesellschaft erfuhr zugleich die Position artikulationsfreudiger Gruppierungen mit starkem Basisrückhalt Unterstützung, für welche die Erhaltung der Regierungsverantwortung und der Regierungsfähigkeit kein Argument allererster Bedeutung mehr zu sein schien, und die insoweit an die vormodernen Positionen der Parlamentskritik anknüpfte. Macht- und stabilitätspolitisch waren solche Erscheinungen unter der breiten Mehrheit der Großen Koalition allerdings belanglos.

2) Die Einnistung des Parlaments in die politische Führung ist von der Rechtswissenschaft frühzeitiger entdeckt worden als von der Politikwissenschaft. Die von Julius Hatschek ausgehende Diskussion kann hier nicht nachgezeichnet werden ${ }^{37}$. Ihre Einsichten hat Ulrich Scheuner schon in der Anfangsphase des Bonner Parlamentarismus dahingehend zusammengefaßt, daß das parlamentarische Regierungssystem gekennzeichnet sei durch die Selbständigkeit der Regierung und die Mitwirkung des Parlaments ${ }^{38}$. Der Boden war also längst bereitet, als Ernst Friesenhahn seine bereits zitierte berühmt gewordene Formel fand und hinzufügte, es sei notwendig und möglich zugleich, „dem Parlament stärkeren Einfluß auf die Führung der Regierungsgeschäfte einzuräumen" 39.1972 hat die Enquetekommission Verfassungsreform Friesenhahns Thesen auch auf die Planung angewandt, wenn auch nur in ihrem Z wischenberich ${ }^{40}$. Vor dem prominenten Forum der Vereinigung der deutschen Staatsrechtslehrer sind Friesenhahns Thesen 1974 eher noch verstärkt und ausgebaut worden: Einer der Berichterstatter schrieb dem Parlament ausdrücklich nicht mehr nur Repräsentation als Funktion zu, sondern Herrschaft ${ }^{41}$.

Idealtypisch ist die Konkretisierung der politischen Führungslinie als Ergebnis eines ständigen Kommunikationsprozesses zwischen Mehrheit und Kabinett zu begreifen. Genau hier setzte die verfassungsrechtliche Diskussion seit Ende der 60 er Jahre an, deren Ziel es war, diese Praxis einer verfassungsrechtlichen Betrachtung zugänglich zu machen, und zwar auf der Basis der dem 20. Jahrhundert entstammenden komplexen Ansätze zum Verhältnis von Parlament und Regierung

37 Siehe Julius Hatschek, Allgemeines Staatsrecht auf rechtsvergleichender Grundlage, Bd. 1, (Leipzig 1909) 48; ders., Deutsches und Preußisches Staatsrecht, Bd. 1 (Berlin 1922) 572-603.

38 Ulrich Scheuner, Der Bereich der Regierung, in: Rechtsprobleme in Staat und Kirche, Festschrift für Rudolf Smend zum 70. Geburtstag (Göttingen 1952) $283 \mathrm{f}$.

39 Ernst Friesenhabn, Parlament (wie Anm. 31) 40.

40 Deutscher Bundestag, Drucksache 6/3829.

41 Thomas Oppermann, Hans Meyer, Das parlamentarische System des Grundgesetzes. Anlage - Erfahrungen - Zukunftseignung, in VVDStRL 33 (1975) 8-68 bzw. 69-119. 
sowie zu beider Partnerschaft bei der Staatsleitung. Damit war gegen die überkommenen Vorstellungen, bei denen sich die Parlamentskritik noch aufhielt, nun endgültig der Anschluß an die verfassungspolitischen und verfassungsrechtlichen Realitäten gewonnen, statt sie schamhaft zu ignorieren oder fälschlich zu exkulpieren oder auch die Flucht in ganz andere Modellvorstellungen anzutreten, wie dies Dolf Sternberger 1964 kritisiert hat ${ }^{42}$.

Kewenig und Bäumlin haben etwa zur gleichen Zeit die frühzeitige Einschaltung der Parlamente in die politische Führung nachgewiesen und gerechtfertigt ${ }^{43}$, wobei sie sogar noch auf der Ebene des Zusammenwirkens der Institutionen Parlament und Regierung verharren. Ihre Thesen beziehen sich noch nicht einmal auf das immer dichter gewordene informelle Beziehungsgeflecht im Schoße der Mehrheitsfraktionen, in welchem parlamentarische Mitbestimmung inzwischen noch viel weiter vorangetrieben worden ist. Alle Mehrheiten in der Geschichte der Bundesrepublik haben diesen Anspruch erhoben, bedingt durch die spezifischen Mehrheitsverhältnisse in den 60er und 70er Jahren sogar in besonderer Weise. Deren Geschichte könnte nicht allein im Blick auf die Kanzlerschaften zutreffend geschrieben werden, sondern nur unter Einbeziehung der wichtigen Fraktionsvorsitzenden Rainer Barzel, Helmut Schmidt und Herbert Wehner. Im Verhältnis zwischen Parlament und Regierung seien Legislative und Exekutive lediglich noch "konstitutionelle Begriffshülsen“, nichtssagend für den gemeinsamen Prozeß der Gestaltung der inneren und äußeren Politik, schrieb später Wilhelm Mössle in seiner rechtswissenschaftlichen Habilitationsschrift mit dem beziehungsreichen Titel „Regierungsfunktionen des Parlaments“44.

3) Die 60er und 70er Jahre waren entsprechend dem öffentlichen Klima auch Jahre der Parlamentsreform, deren Ziel es vorrangig gewesen ist, die im Selbstverständnis der Abgeordneten verankerte Teilhabe an der Staatsleitung konkret zu unterstützen. So lang und beeindruckend die Liste der einzelnen Maßnahmen ist $\mathrm{t}^{45}$, so sehr bedurfte es doch auch eines inneren Klimawandels, um Reformprozesse anzustoßen. Bis 1961 ist der Bundestag bei geringer Fluktuation des Abgeordnetenpersonals hinsichtlich seiner parlamentarischen und historischen Erfahrungen relativ homogen gewesen. Zugleich herrschte in den Führungsämtern, denen Apparat und Ressourcen zugeordnet waren, geringe Rotation. Mit dem Einzug jüngerer Abgeordneter, die von neueren Erfahrungen geprägt und vor allen Dingen aus ihrer Berufswelt gänzlich andere betriebsorganisatorische Bedingungen gewohnt waren, stellte sich nach den Wahlen von 1965 die Frage nach den Arbeitsbedingungen mit Schärfe. Sie führte zu innerparlamentarischer Kritik und

42 Dolf Stemberger, Parlamentarische Regierung und parlamentarische Kontrolle, in: PVS 5 (1964) 6-19.

43 Richard Bäumlin, Die Kontrolle des Parlaments über Regierung und Verwaltung, in: ZSR 85 (1966) 165-319; Wilbelm Kewenig, Staatsrechtliche Probleme parlamentarischer Mitregierung am Beispiel der Bundestagsausschüsse (Bad Homburg u.a. 1970).

44 Wilhelm Mössle, Regierungsfunktionen des Parlaments (München 1986) bes. 187.

45 Siehe Peter Schindler, Datenhandbuch zur Geschichte des Deutschen Bundestages, Bd. 2 (Baden-Baden 1999) 2856-2872 (für die 5. bis 8. Wahlperiode = 1961-1980). 
löste Veränderungen und Verbesserungen aus. Als Hemmnisse empfand eine Vielzahl von Abgeordneten die positionellen Interessen der Regierung und des eigenen Establishments, zugleich aber auch die mangelnde Unterstützung der Öffentlichkeit, die im wesentlichen von zwei Strömungen geprägt gewesen ist: Gleichgültigkeit einerseits, praxisferne oder gar antiparlamentarische Fundamentalkritik andererseits. Dennoch wurde diese Kritik nach der von den Abgeordneten selbst empfundenen dringenden Notwendigkeit $(52,7 \%)$ und ihrer allgemeinen Stimmung $(44,6 \%)$ als drittstärkster Stimulus $(30,4 \%)$ für ein Reformklima genannt ${ }^{46}$.

Um diese Öffentlichkeit hat der Bundestag kontinuierlich geworben. Sie besser zu erreichen, war seit jeher eines der beiden großen Ziele von Reformanstrengungen. Auch in der Periode zwischen 1960 und 1980 wurde der „Verlebendigung“ der Debatte, der Verbesserung ihres Stils und ihrer Struktur erhebliche Aufmerksamkeit geschenkt - wie überhaupt der Stärkung der Öffentlichkeitsfunktion, z.B. durch die Einführung der Aktuellen Stunde und der Kabinettsinformation sowie durch die fakultative Öffentlichkeit von Ausschußsitzungen. Die zweite Zielrichtung war speziell in dieser Phase die Verteidigung und Verbesserung von Entscheidungskompetenz und Konkurrenzfähigkeit der Abgeordneten und Fraktionen gegenüber den anderen Akteuren im politischen Entscheidungsprozeß. Entsprechende Maßnahmen umfaßten die Bereitstellung von Büroraum für die Mandatare (Bau des „Langen Eugen“), Verbesserungen ihrer Arbeitsbedingungen sachlich wie personell sowie vor allen Dingen die Erschließung der Möglichkeiten der elektronischen Datenverarbeitung für das Parlament. Seinem Selbstverständnis und der Tradition gemäß hat der Bundestag beides versucht: sich sowohl als Redeparlament zu profilieren als auch als Arbeitsparlament zu bestehen. Dabei blieb ihm immer bewußt, daß seine Mitregierungskompetenz weniger von rhetorischen Glanzleistungen und öffentlicher Resonanz abhängt, sondern von seiner politischen und sachrationalen Expertise.

In diesem Kontext sind jenseits der Details der Reform- und Revisionsbeschlüsse von 196947 und $1980^{48}$ drei die parlamentarische Leistungsfähigkeit abstützende Entwicklungen ausschlaggebend gewesen: a) die Herausbildung eines sich stark differenzierenden Systems beratender Entscheidungsvorbereitung, das im Ausschußwesen an ältere Traditionen anknüpft. Neuer und vergleichsweise unbekannt geblieben ist die arbeitsteilige Organisation der Fraktionen in den 60er Jahren. Es begann auch die institutionalisierte Heranziehung außerparlamentarischen Sachverstands durch die Einrichtung von Enquetekommissionen und die verstärkte Nutzung von Hearings, die vorher boykottiert worden waren; b) der pragmatische, in den 70er Jahren aber zielbewußt vorangetriebene Aufbau eines Mitarbeiterstabs beim Gesamtparlament, bei den Fraktionen und beim einzelnen Abgeordneten. Dieser Stab war zunehmend zu qualifizierter wissenschaftlicher

46 Hans Maier u.a., Parlament und Parlamentsreform (wie Anm. 7) 51-76, bes. 61.

47 Peter Scholz, Bilanz der Parlamentsreform '69, in: Heinrich Oberreuter (Hrsg.), Parlamentsreform in westlichen Demokratien (Passau 1981) 157-171.

${ }^{48}$ Joseph Bücker, Geschäftsordnungskorrekturen - Die Revision 1980, in: ebd. 173-181. 
Zuarbeit in der Lage und konnte sich immer stärker auf eine ständig ausgebaute und verbesserte Informations- und Dokumentationsbasis stützen; c) speziell durch den Generationenwandel die Herausbildung eines neuen Abgeordnetentyps, der mit einer derartigen Infrastruktur tatsächlich umzugehen weiß und an den traditionellen Honoratiorenabgeordneten kaum mehr erinnert.

Reformmaßnahmen, die im Parlament selbst hohe Zustimmung finden, liegen ausnahmslos in diesem Bereich. Angesichts seiner Bedeutung wäre es völlig unangemessen, ihn lediglich als betriebsorganisatorisch abzutun, so pragmatisch die einzelnen Schritte auch Zug um Zug vorangetrieben worden sein mögen. Strukturelle Reformen, die das System in andere Richtungen - etwa des idealtypischen britischen oder amerikanischen Modells - gedrängt hätten, sind im Bundestag nie populär gewesen.

Trotz aller Bemühungen sind die Transparenz- und Kommunikationsdefizite nie behoben worden. Sachrationalität in hochkomplexen Materien der Gesetzgebung schränkt Öffentlichkeitsattraktivität unweigerlich ein. Je mehr er rationale Problemlösungskompetenz in diesen $20 \mathrm{Jahren}$ untermauert hat, umso mehr fielen legislatorische Leistungsentfaltung und Legitimationsleistung des Bundestages auseinander. Diese Diskrepanz erscheint unvermeidlich. Aber sie beschwört die Gefahr herauf, daß Leistungen, die für die Akzeptanz der parlamentarischen Demokratie Bedeutung hätten, im Legitimationsprozeß nicht fruchtbar werden. Damit laufen sie letztendlich leer. Doch kein Reformprozeß vermochte bisher das Parlament aus diesem Dilemma zu befreien. Selbstverschuldet sind dagegen kommunikative Defizite gegenüber gesellschaftlichen Wandlungsprozessen, neuartigen Problemstellungen und Gruppen, die sich um diese herum kristallisieren. Solche Defizite sind nicht durch Reformen, sondern nur durch Sensibilität und Responsivität zu vermeiden. In den 70er Jahren haben die Abgeordneten es daran fehlen lassen und dieses Versäumnis prompt mit einer sektoralen Legitimitätskrise und der Etablierung einer neuen Partei mit der Folge neuer parlamentarischer Konkurrenz quittiert. Aber diese Integration einer sich selbst definierenden "Anti-Parteien-Partei" mit ursprünglich durchaus antiparlamentarischen Tendenzen $^{49}$ in das System der parlamentarischen Demokratie weist auf dessen Offenheit, Anpassungs-, Absorbtions- und Integrationskraft hin.

${ }^{49}$ Heinrich Oberreuter, Abgesang auf einen Verfassungstyp? Aktuelle Herausforderungen und Mißverständnisse der parlamentarischen Demokratie, in: APuZ 2 (1983) 19-31. Die Positionen sind in zwei Editionen übersichtlich dokumentiert: Bernd Guggenberger, Claus Offe (Hrsg.), An den Grenzen der Mehrheitsdemokratie (Opladen 1984) und Heinrich Oberreuter (Hrsg.), An den Grenzen der parlamentarischen Demokratie (München 1986). Die Angriffe gegen „Stellvertreterpolitik“ und "Mehrheitsdemokratie“ in den 80er Jahren, die auf eine „neue Form“, auf "grundsätzliche Veränderung“ des parlamentarischen Systems abzielten, bedienten sich bei praktisch identischer Zielsetzung aus dem gleichen Arsenal wie die Systemkritik der 68er. Da auch schon Rudi Dutschke der revolutionären Avantgarde der Intellektuellen selbstlegitimierte Führung zugeschrieben hatte, war die später postulierte Legitimation durch Wahrheit (statt Mehrheit) und höheres Bewußtsein substantiell nicht neu, höchstens deutlicher. 


\section{Bilanz}

Was hat die Parlamentsreform geleistet? Eine Bilanz ergibt, daß sie von der funktionalen Kritik ausgehend seit den 60er Jahren und seither fortgesetzt beständig zahlreiche zeitgemäße Antworten gegeben hat, welche die eingeschlagene Praxis parlamentarischer Mitregierung unterstützten. Auf die antiquierte Modernitätskritik wurde höchstens marginal sowie gemäß den Anstößen und im Rahmen der Vorgaben des Bundesverfassungsgerichts reagiert, welches der exzessiven Entwicklung zum Fraktionenparlament durch die Verteidigung essentieller Rechte des einzelnen Abgeordneten zur Mitarbeit in den Ausschüssen und im Plenum gewisse Grenzen gesetzt hat, ohne den generellen Trend auch nur im geringsten in Frage zu stellen. Die Systemkritik ließ der Bundestag gänzlich unbeachtet - ganz zurecht; denn aus ihr hätte er keine Anstöße zu seiner Stärkung, sondern nur Hinweise zu seiner substantiellen Schwächung, wenn nicht sogar Überwindung erfahren können.

1) Über die Jahrzehnte hinweg hat sich die Parlamentsreform als steter Prozeß der Angleichung von Mitteln und Möglichkeiten zu Kontrolle, Mitgestaltung und Mitregierung etabliert. Er vollzog sich auf Geschäftsordnungsebene, im Bereich der Arbeitsorganisation und Arbeitsmittel und - von der Institution am wenigsten beeinflußbar - auf der Ebene des Abgeordnetenpersonals, dessen Erfahrungen, Qualifikationen und Ansprüche sich gewandelt haben. Es hätte wenig Sinn gemacht, die Instrumente zu modernisieren, wenn das Personal nicht die Fähigkeit gehabt hätte, mit ihnen auch umzugehen ${ }^{50}$. Dieser Reformprozeß in Permanenz kreierte zum einen immer wieder Einzelschritte, zum anderen aber Überlegungs- und Maßnahmepakete, die durchaus Akzente setzten.

1969 stand die Arbeitsfähigkeit des einzelnen Abgeordneten und der Fraktionen im Zentrum, umlagert von wichtigen prozeduralen Verbesserungen wie z.B. dem Selbstbefassungsrecht der Ausschüsse und ihrer potentiellen Öffentlichkeit. Strukturelle Überlegungen, die freilich nicht zu Ende gedacht und im Diskussionsprozeß eher wieder zurückgeführt worden sind, ergaben sich in den 70er Jahren ${ }^{51}$ im Rahmen der Enquetekommission Verfassungsreform, die z.B. bei der Bundestagswahl an wenigstens begrenzt offene Listen nach bayerischen Vorbild dachte, um den Einfluß der Bürger zu erhöhen und auch den Bundestag als Gesetzgeber durch Verfahrensvereinfachungen - z.B. italienisches Ausschußmodell, verstärkte Verordnungsermächtigungen - zu entlasten. Nichts davon ist realisiert worden: Zum einen, weil sich die Parteiführungen ihren Einfluß auf die Kandidatenauswahl nicht aus der Hand nehmen lassen wollten, zum anderen, weil tiefver-

50 Kritik an der Rekrutierung hat ihren Platz bei in späteren Jahren zugespitzten Tendenzen zur Karrierisierung und Professionalisierung der Abgeordnetenlaufbahn. Dazu Dietrich Herzog, Politische Karrieren. Selektion und Professionalisierung politischer Führungsgruppen (Opladen 1975).

51 Vgl. Carl Otto Lenz, Gerald Kretschmer, Strukturfragen - Die Alternativen der EnqueteKommission Verfassungsreform, in: Heinrich Oberreuter (Hrsg.), a.a.O. (Anm. 47) 183-200. Siehe auch den Schlußbericht Deutscher Bundestag, Drucksache $7 / 5924$. 
wurzeltes Parlamentsverständnis und historische Erfahrungen dem entgegenstanden. Im Jahre 1980 erfolgte eine Geschäftsordnungsreform mit funktionslogischem Ansatz. Den strukturellen Vorgaben des parlamentarischen Regierungssystems sollte besser entsprochen werden können, als es im Rahmen eines weithin noch von konstitutionellen Vorgaben geprägten Normengefüges möglich war. Terminologisch kam das nicht zum Ausdruck, doch war diese Geschäftsordnungsrevision von zwei Leitlinien geprägt: Zum einen sollte die Mehrheit nicht daran gehindert werden, ihre Funktionen und Aufgaben sachgerecht wahrzunehmen, zum anderen sollte auch die Opposition mit unabdingbaren Rechten ausgestattet werden, um ihre Funktion in der parlamentarischen Auseinandersetzung zu erfüllen ${ }^{52}$. Sie hatte übrigens schon seit 1978 bei der Finanzausstattung der Fraktionen zur Verstärkung der Chancengerechtigkeit einen Oppositionsbonus erhalten.

2) Den Parlamentariern ist stets selbst bewußt gewesen, daß sie Reform in kleinen Schritten betreiben. Ein großer Wurf ist ihnen nie gelungen. Sie haben ihn auch nicht angestrebt. Er wäre auch zum Scheitern verurteilt: Institutionen sind nur in Grenzen und nur im Rahmen des Überschaubaren und Kalkulierbaren zu reformieren. Ziel, Ende und Ratio müssen einsichtig, verläßlich und nachvollziehbar sein. Sodann ist das weit verbreitete machtopportunistische Kalkül zu beachten. Mehrheiten wollen die Beherrschung von Institution und Verfahren, die sie ohnehin mit der Minderheit teilen müssen, nicht gänzlich aus der Hand geben und unüberschaubare Risiken eingehen, wie auch die Frontbench in der regierenden Mehrheit ebenso wie in der Opposition naturgegeben skeptisch bleibt gegenüber jeder Gefährdung ihrer Privilegien. Schließlich bedürfen Reformen der Institution und der Prozeduren der parlamentarischen Demokratie, deren Integrität ja bewahrt werden soll, eines parteiübergreifenden Konsensus. Als Ergebnis solcher Voraussetzungen kann sich keine große Reform einstellen, sondern in aller Regel höchstens ein mittlerer gemeinsamer Nenner.

Der Bundestag hat in seinen Reformprozessen funktional gedacht und sich nicht von dem Motto „mehr Demokratie wagen“ zu Experimenten verführen lassen. Seine Reformschritte blieben, um den Jargon der APO aufzugreifen, ,inkrementalistisch". Wenn sich die parlamentarische Demokratie im Spannungsfeld von Effizienz, Transparenz und Partizipation entfaltet, dann hat sich der Bundestag akzentuiert für Effizienz entschieden und sich damit durchaus auf die Seite seiner Wähler gestellt. Denn diese sind ausweislich ihrer sich immer stärker steigernden Volatilität weniger am Input als am Output, also an den erfahrbaren Leistungen, der parlamentarischen Demokratie interessiert.

3) Die Herausforderungen durch ihre Kritiker kann die plurale parlamentarische Demokratie, wie sich zwischen 1960 und 1980 durchaus zeigte, ruhig annehmen. Denn sie ermöglicht im Rahmen rechtsstaatlicher Kompetenzordnung und Verfahrensregeln konkurrierenden Interessen und Wertvorstellungen Zugang zum Entscheidungsprozeß. Damit gewinnt das politische System nicht nur einen

52 Joseph Bücker, a.a.O. (Anm. 48). 
höheren Grad an Funktionalität; auch gegenüber den Verwirklichungschancen politischer Freiheit durch seine auf Machtkontrolle, Konsensbeschaffung und Legitimitätssicherung angelegten Repräsentationstechniken und Willensbildungsmechanismen bedeuten die Alternativangebote der plebiszitären Demokratie und des Rätesystems eine immense Regression. Der moderne Parlamentarismus ist ihnen im Leistungs- wie im Legitimationszusammenhang des politischen und sozialen Systems überlegen.

Seine wirklichen Herausforderungen liegen nicht in der Kritik, sondern in den sich rasch verändernden und immer komplexeren Problemstellungen auf der zentralen Entscheidungsebene, im Durchsetzungsanspruch demokratisch nicht legitimierter Teilgruppen auf Kosten der Allgemeinheit und im Autonomieanspruch von Subsystemen ${ }^{53}$. Die Antwort darauf liegt gleichbleibend in der Stärkung des Parlaments als zentraler, demokratisch legitimierter und verantwortlicher Institution durch Reformen, welche seine formale Entscheidungskompetenz untermauern - einerseits sachrational durch die Verbesserung seiner technisch-wissenschaftlichen Infrastrukturen, andererseits politisch durch adäquate Organisation und Funktionssicherung der systemimmanenten Legitimationsprozesse.

Durch Parlamentsreform allein ist dies nicht zu leisten und auch in den 20 Jahren zwischen 1960 und 1980 nur in Ansätzen befördert worden. Politische Kultur, historische Traditionen, parlamentarischer Stil, verfassungspolitischer Konsens sind in diesem Kontext zu beachtende Faktoren. Insofern sind auch nach 1980 Aufgaben zur Positionsverteidigung des Parlaments in der Staatsleitung übriggeblieben und neue hinzugekommen, die sich nicht zuletzt durch die Politikverflechtung im Föderalismus, durch die Europäisierung politischer Entscheidungsprozesse und schließlich auch durch die Priorität televisionärer Politikvermittlung stellen. Diese Herausforderungen sind von neuer Qualität, die durchaus die Frage rechtfertigt, inwiefern jenseits seiner formalen Zuständigkeiten der Bundestag noch immer ein wichtiges und kompetentes Zentrum politischer Entscheidung, Aufmerksamkeit und Legitimation geblieben ist. Währenddessen verharrte die Parlamentskritik im Rahmen des Überkommenen.

53 Die in der föderalistischen Struktur der BRD und in der Supranationalisierung (europäische Integration) begründeten Probleme haben sich damals schon herausgeprägt - später zugespitzt. 